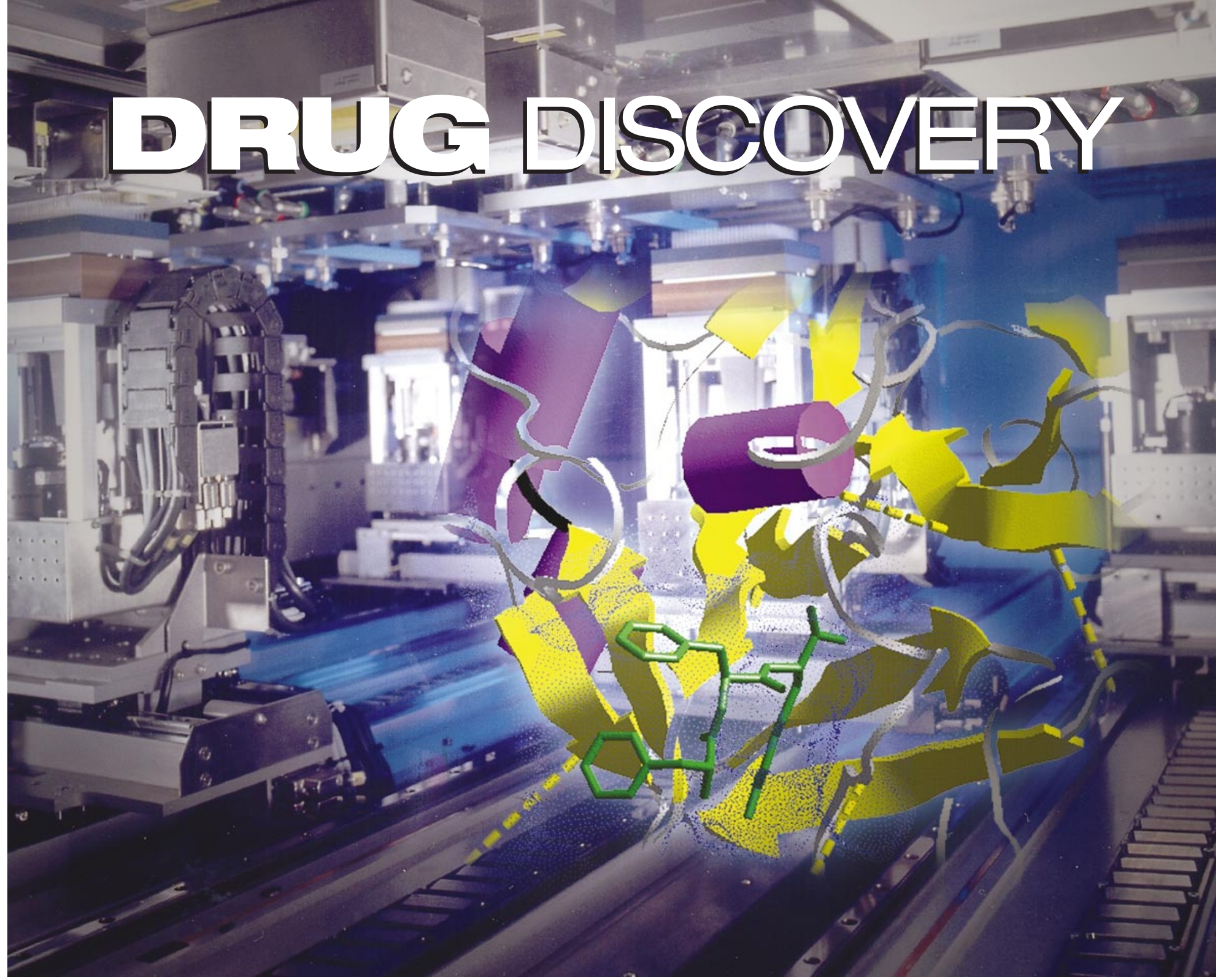

SCREENING FOR DRUG DISCOVERY

453 The leading question

AUTOMATING THE SCREENING PROCESS 453

GETTING TO KNOW THE FAMILY

455

HOW SMALL SHOULD YOU GO?

457

FRAGMENTING THE PROBLEM

459

TABLE OF SUPPLIERS

461 


\section{Screening for drug discovery: The leading question}

A Il pharmaceutical researchers know the feeling. Somewhere out there must be that elusive molecule - one that will inhibit this enzyme or activate that receptor in the way they want, and without causing unwanted side-effects. But finding it is another matter. For small-molecule drugs - the mainstay of the pharmaceutical industry - time-consuming and expensive screening is needed to pick out promising candidates from the vast number of natural and synthetic compounds available. Testing large numbers of compounds to see if they produce an appropriate biochemical or cellular effect is usually one of the first steps in the drug-discovery pathway, and ways of making this screening faster, more effective and less expensive are in continual development.

A positive response in a first round of screening in a biochemical assay identifies the primary 'hit' compounds. These molecules then go into more screens to see if they have physicochemical and pharmacological properties that are not too incompatible with making a drug - if it passes this filter, a hit becomes a 'lead'.
Lead compounds then undergo further rounds of chemical refinement and biological screening before finally entering clinical testing. With a good deal of luck, your lead might eventually be approved as a drug 12-15 years after testing began.

But all is not quite as it should be in the drug industry. Estimates vary, but in general analysts agree that each major pharma company needs to launch three or four new products a year in order to sustain the present level of growth. A glance at the chart on the right shows that productivity over the past few years has been well below this level, with the top 20 pharma companies averaging just over one new launch a year.

Increasing the number of leads is thus high on the drug-discovery agenda.

Foreseeing the coming deficit, companies implemented a number of strategies in the late 1980s intended to do this.

Combinatorial chemistry was used to generate larger libraries of compounds for testing, and high-throughput technology, including increasing miniaturization and automation, was deployed to screen these

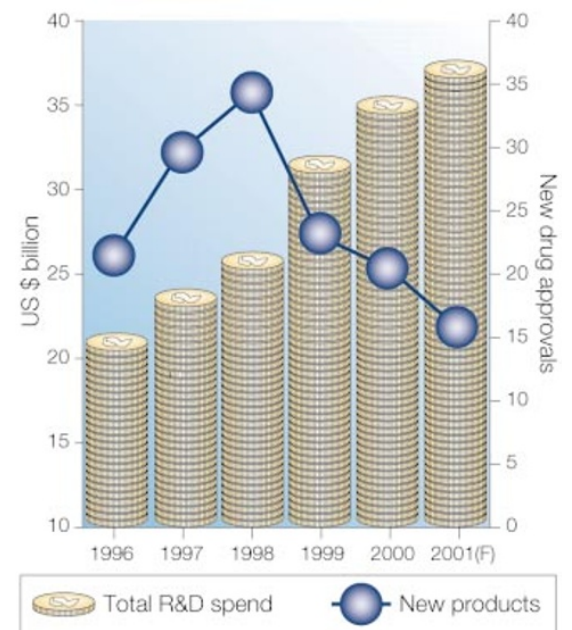

R\&D spend versus new drug launches by the top 20 pharma companies.

libraries more rapidly. But despite tremendous advances in all aspects of the screening process, chiefly the increased use of automation, these improvements did not bring about the expected rise in productivity, and the industry's drug pipelines still look decidely thin.

Advocates of high-throughput screening claim that the technique is still in its infancy. "High-throughput screening is not 100,000 tests a day, it's 100,000 tests every day," says

\section{AUTOMATING THE SCREENING PROCESS}

Many companies are finding that installing an efficient highthroughput screening facility is as much about introducing new methods of workflow management as it is about getting the technology right. A single screen involves about 80-100 separate activities, such as making sure each reagent is available, producing recombinant protein and plating the compounds. A major pharmaceutical company could be doing 50-100 screens a year, 40-50 of which may be running concurrently. To keep these running efficiently needs collaborative planning and a commitment to an effective supply chain.

"If the plates don't turn up on the Monday morning, your screen just isn't going to get done. This can't be planned in a lab book," says Richard Archer, chief executive of The Automation Partnership, a company based in Royston, UK, which supplies high-throughput screening equipment. But the basics of supply-chain management do not come easily to scientists, says Archer, as they tend to get excited by the one novel assay they're planning, rather than by the prospect of keeping the more conventional assays running.

Another difficulty in implementing high-throughput screening is the bias that has existed when it comes to recruiting 'screeners' as opposed to research-focused PhDs. "You get higher up the tree by being a bright scientist than being an efficient process manager," says Mark Beggs, head of consulting

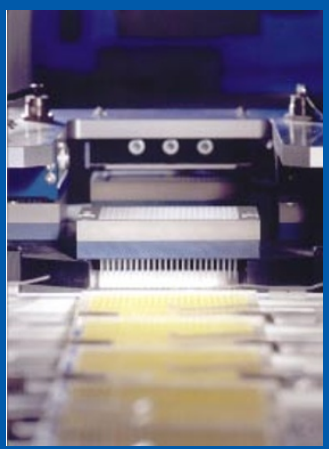

Tip washing in TAP's Asset screening platform at The Automation Partnership. "The person who discovers the kinase will get further than the one who instigates highly productive screens to identify inhibitors for it."

Despite these hurdles, process management is increasingly seen as being key to successful screening. Equipment manufacturer Amersham Biosciences, based in Piscataway, New Jersey, recently struck a deal with Cimarron Software, Inc, a laboratory workflow management consultancy based in Salt Lake City, Utah. And when biotech firm Amgen set up a screening facility at its headquarters in Thousand Oaks, California, it brought in a strong engineering team of technical support staff to keep the equipment working.

"It seemed to them to be the obvious thing to do, and the firm was surprised to find that this was fairly unique in the industry," says Archer. 
Richard Archer, chief executive of The Automation Partnership in Royston, UK, a company that makes automated equipment for screening. "You can't say that automated high-throughput screening doesn't work, because nobody is doing it yet."

But all now agree that for screening, quality is more important than quantity. Throughout the industry the emphasis is shifting - from screening the greatest number of compounds as quickly as possible, to making sure that high-quality compounds are going into robust and reproducible assays, and to understanding potential targets better (see "Getting to know the family", below).

\section{Getting organized}

The basic workhorse of screening is the microtitre plate, and a number of companies have developed robust, automated screening platforms based on this format. Initially, the plates featured 96 wells, which allowed the same number of different molecules to be screened for a given activity. More recently, the miniaturization of the simpler types of assay has seen the 384-well plate become standard, leaving those with 96 wells to be used in more complicated assays.

Plates with an even higher capacity (1,536 and even 3,456 wells) are used more rarely because the problems associated with handling minute volumes can add significantly to the cost of the assay (see

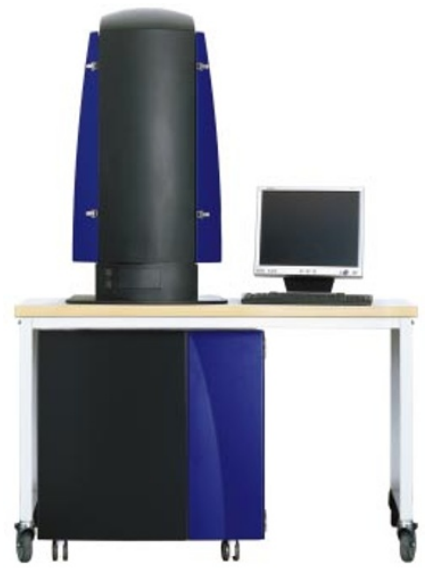

Portable screening: Amersham's LEADseeker

"How small should you go?", page 457). Amersham Biosciences, an equipment manufacturer based in Piscataway, New Jersey, is one of several companies producing the new generation of robust screening platforms. Its LEADseeker, for example, is designed for decentralized primary screening. It uses imaging technology based on a charge-coupled device that detects fluorescence and luminescence, and allows a whole 96-, 384or 1,536-well plate to be read at a time.

Amersham sees the LEADseeker as a step on from earlier technologies based on tracking radiolabelled samples, such as scintillation proximity assays. Indeed, a general feature of the new generation of equipment is that it uses fluorescencebased assays. These have high signal-tonoise ratios, and therefore offer higherquality data compared with radioactivitybased assays - so much so that in many cases signal detection is so clear there is no need to do replicate wells.

But supplying the equipment is just one part of the equation. Manufacturers also recognize that organizing the highthroughput laboratory's workflow is equally important (see "Automating the screening process", page 453). "Different companies want different things," says Mike Evans, vice-president for bioassays at Amersham. "Some want 'turnkey solutions', whereas others want to mix and match with piecemeal technology."

Making products tailored to an individual user's requirements is also becoming a common theme among providers of software and bioinformatics solutions for screening. Here, the main cry from the industry is for data-handling packages that conform to common standards so that they can be interfaced with existing systems. "In the past, software companies were sometimes guilty of trying to impose their own standards on the industry," says Scott Kahn, a senior vicepresident at Accelrys, a software manufacturer in San Diego, California.

Accelrys is one of several companies that favour the development of generally

\section{GETTING TO KNOW THE FAMILY}

Most companies seek hits against members of the main families of proteins known to be likely drug targets, such as G-proteincoupled receptors, kinases and proteases. Not surprisingly, achieving selectivity for just one member of such a family can be a considerable challenge.

"Just about anybody can get a hit against a kinase," says Richard Scott, head of chemoinformatics at De Novo

Pharmaceuticals, a company based in Cambridge, UK, that uses virtual screening to discover drug leads. "It's easy to get a hit, but not nearly so easy to get a selective hit."

As a result, many companies are striving to get to know their target protein families better. This approach has always been central to the philosophy of Vertex Pharmaceuticals, a drugdiscovery firm in Cambridge, Massachusetts, but Mark Namchuk, head of high-throughput screening at the company, says that this is now a trend throughout the industry. At Vertex, early discovery is focused on whole protein families rather than individual target proteins.

Emphasizing just how central an understanding of the biology of the target family is to the usefulness of the screening data, the high-throughput screening facility at Vertex is run as a division of the enzymology group, which refers to screening as "highthroughput enzymology", and to screening campaigns as "experiments". As well as giving valuable insights into selectivity, "this approach allows you to view today's data as foundations for

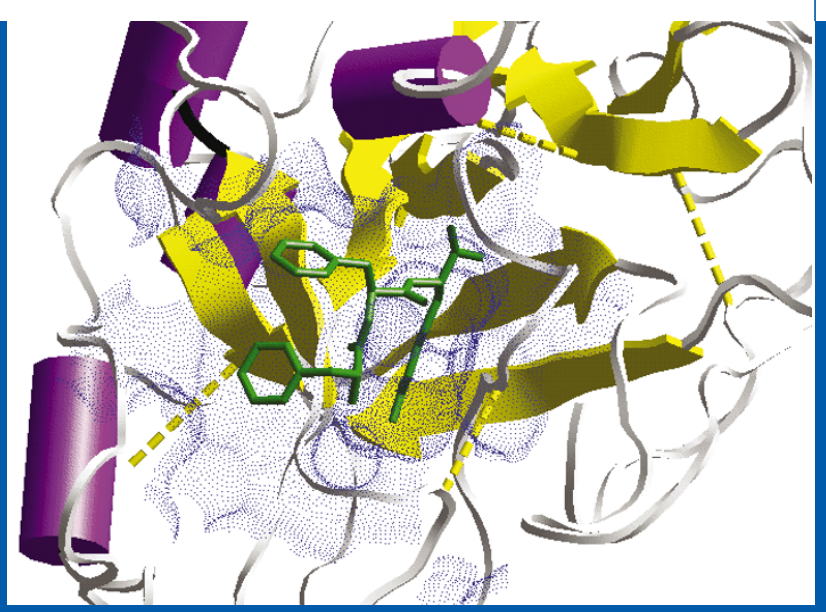

future projects", Namchuk says.

Although not yet a replacement for bioassaying the activity of your molecule on the proteins themselves, virtual screening of similar targetrs can help in identifying problems that might crop up further down the pipeline. "Virtual screening can give you a heads-up to other potential interactions," says Scott Kahn, a senior vice-president at software manufacturer Accelrys in San Diego, California. 
recognized external, non-proprietary standards. The company uses Microsoft standards, as does its main competitor, Spotfire in Somerville, Massachusetts. This offers the end-user additional benefits. "The fact that both companies are developing to a common standard means that although they're competing head-tohead, users can integrate their products however they wish," says Kahn.

\section{The shape of things to come}

Libraries of small-molecule compounds are the raw material that goes into the primary screens. Although there is general agreement about how assay platforms should be developing, there seems to be little consensus about the shape of the ideal compound library. Opinions vary on how big a library should be, and how companies should design, store and handle its contents.

One idea that is exciting interest is to profile and filter compounds for drug-like properties such as solubility and lipophilicity before they ever get into the library. This should give medicinal chemists an easier time by ensuring that lead compounds need less refinement to turn them into drugs.

Companies such as Argenta Discovery, a medicinal chemistry design and screening company based in Harlow, UK, are now screening compounds for a range of drug-like behaviours before they enter the company's libraries. Chris Newton, chief scientific officer at Argenta, describes the profiling as "multi-parametric optimization”.

Early whittling away of compounds with undesirable properties can also be done by computer, and in silico screening for 'drug-likeness' is a central component of the 'virtual-screening' strategies of companies such as Argenta, De Novo Pharmaceuticals in Cambridge, UK, and Vertex Pharmaceuticals in Cambridge, Massachusetts. "We're trying to encode the common sense of medicinal chemists into the computer," says Mark Namchuk, head of high-throughput screening at Vertex. The company uses a proprietary program called REOS (rapid elimination of swill) to eliminate non-drug-like molecules before compounds make it through to the primary screen.

It is too early to judge the success of virtual-screening programmes, but two independent teams of researchers, from Merck laboratories in Rahway, New Jersey, and from Brian Shoichet's group at Northwestern University, have shown structure-based computational docking used as a filter can hugely enrich the hit rate compared with random screening.

\section{Compounds on display}

One area of chemical screening where the drive towards automation has been somewhat weak is compound handling.

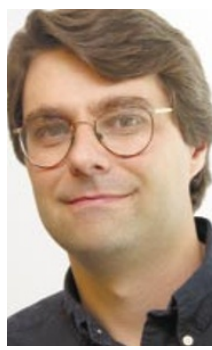

Namchuk:

screens are

experiments.
The preparation of microtitre plates placing the various compounds into their appropriate wells ready for screening — is still relatively slow. Graffinity

Pharmaceuticals, a drug-discovery company based in Heidelberg, Germany, has come up with an alternative strategy. It

sprays 10,000 compounds as spots onto a 'chip', and their affinity for a target protein can be read simultaneously by an imager based on the surface plasmon resonance method developed by equipment manufacturer Biacore in Uppsala, Sweden (see "Fragmenting the problem", page 459).

Graffinity's early microarrays were made up of binary combinations of monomers using amide coupling, as these are easy to make and can rapidly generate a large library of compounds. The company now has a more diverse library of 70,000 compounds presented on microarrays. These can be screened against a protein target in a day, requiring just $5 \mathrm{mg}$ of protein.

This microarray platform generates a relatively high number of hits, but many of them will be for compounds with similar structures, because the screen

\section{HOW SMALL SHOULD YOU GO?}

Miniaturization has been one of the triumphs in screening technology over the past decade or so, mainly because of advances in the automation of liquid handling, control software and detection systems. A few examples of ultra-high-throughput screening already exist, with Vertex in San Diego, California, for instance, performing the majority of its assays using 3,456-well microplates, in which each assay is done in a volume of just $1 \mu \mathrm{l}$.

Affymax, a drug-discovery firm based in Palo Alto, California, is reported to be working on a 20,000-well plate in which each well would have a volume of just $25 \mathrm{nl}$. And although most of the endeavour has been directed towards miniaturizing microplates, other ultra-high-throughput formats are being developed, the screening of microbead-attached combinatorial libraries, for instance, by companies such as Luminex in Austin, Texas, and Illumina in San Diego, California.

Although these examples are undoubtedly a taste of the future, miniaturization is not for everyone, and does not suit every purpose. Except when compound or protein amounts are critical factors, miniaturization is unlikely to make a big difference to the efficiency of primary screening, although doing assays in less time makes it easier to keep conditions standardized.

For Vertex's cell-based assays, the transition from 384- to 3,456-well plates meant that the number of cells needed dropped from $10,000-40,000$ per well to about 200 . This means that the company can work with cell types that are relatively difficult to get hold of, such as disease cells, says Paul Negulescu (below), vicepresident of discovery biology at Vertex. In principle, 3,456-well plates could be used to study single cells, but at that level the cells' 'individuality' starts to become a problem, giving uneven responses and so degrading the data.

Vertex's 3,456-well Nanowell plates enable ultra-high throughput.
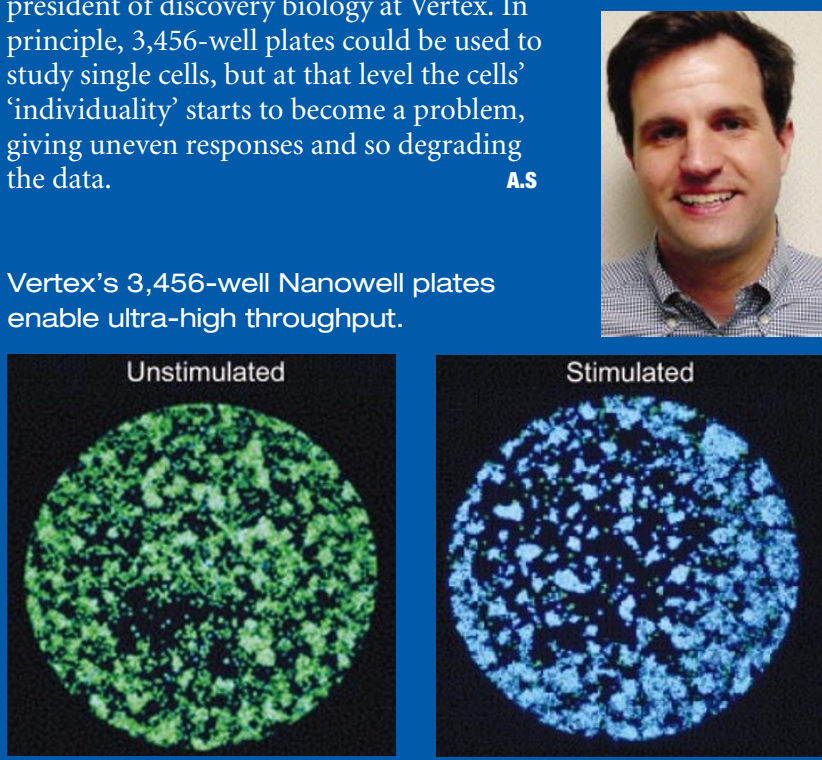
picks up the activities of the monomer building blocks as well as the binary combinations.

\section{High-content screening}

The amount of information that can be gleaned from a screen can be increased by using cell-based systems. Screens such as those offered by Amersham Biosciences, Evotec OAI in Hamburg, Germany, and Vertex Pharmaceuticals in San Diego, California, allow complex biological data on lead-compound behaviour to be collected.

"Although the industry has been doing in vitro assays for a long time, there is a big increase in complexity when you start thinking about using whole cells," says John Anson, vice-president of systems development at Amersham. For instance, instead of just measuring the binding of a ligand to a receptor in vitro, you might now need to track the movement of a labelled molecule from the cytoplasm to the nucleus. Researchers are also beginning to measure more than one event at a time, for instance by using two different reporter molecules, and this is adding to the complexity.

The increased intricacy of assay systems is changing perceptions of the screening process. "The ability to track the internalization or translocation of a cellular component allows you to think more deeply about what you want to get out of a screen," says Paul Negulescu, vice- president of discovery biology at Vertex.

Although most researchers would admit that a degree of serendipity operates in screening for hits and leads, most screens are hypothesis-driven, using assays designed to test the effects of compounds on a particular protein target. But CombinatoRx, a two-year-old company based in Cambridge, Massachusetts, has taken a very different approach. It screens binary combinations of existing drugs to see whether drugs that have known effects when acting singly might have different, unexpected, effects when used in combination.

\section{Double value}

Most drugs do not, in fact, target single proteins, explains the company's chief executive, Alexis Borisy. Instead they interact with a number of targets at a variety of potencies.

"Recognizing the inherent complexity of biological systems, we want drugs that will interact with multiple points in a pathway, rather than the 'sledgehammer' strategy of affecting just one key protein," he says, reversing the usual mantra that drugs should be as selective as possible.

The data generated by CombinatoRx's screens are built into 'interaction spaces' to illustrate the dose-response relationship of the two drugs in combination. At present, the company has
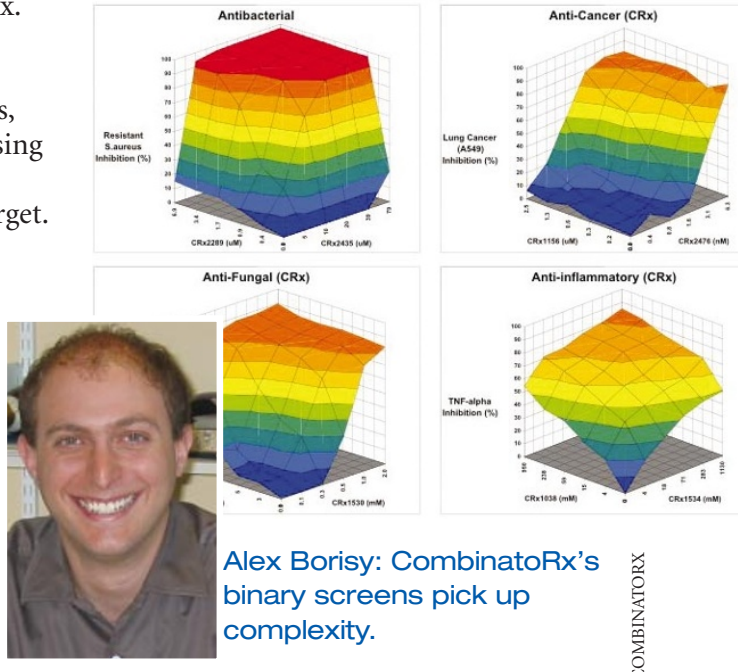

Alex Borisy: CombinatoRx's binary screens pick up complexity.

a screening library of 12.5 million binary combinations. And because all these molecules have already been approved by the US Food and Drug Administration, and are mostly off-patent, it should be possible rapidly to develop any hits for further testing. CombinatoRx plans to start clinical trials on its first sets of binary combinations later this year.

For all these new approaches to screening, the number of new compounds entering clinical trials in the coming years will be the ultimate measure of their success.

Adam Smith is editor of Nature Reviews Drug Discovery.

\section{FRAGMENTING THE PROBLEM}

Bioassay-based screening approaches search for hits with, for example, inhibitor constants at least in the low micromolar range. To get this much potency, a minimum number of interaction points between the molecule and the target protein are needed, which means that only larger compounds generate an initial hit.

Over the years, the compounds held by drug-discovery companies in their collections have been getting bigger, as screeners and medicinal chemists have chased the goal of potency. But this brings its own problems. "It is generally accepted that

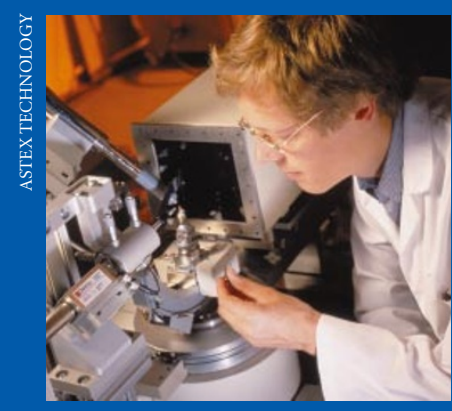

Astex is building leads with X-ray crystallography. larger compounds lead to more late-stage attrition," explains Harren Jhoti, cofounder and chief scientific officer of Astex Technology, a lead-discovery company based in Cambridge, UK. "Chemically refining large initial hits to work out which groups are important or not can be very time consuming." The highly sensitive screening techniques that are now emerging, such as nuclear magnetic resonance and surface plasmon resonance, are providing the opportunity to search for lower-affinity hits, in the high micromolar or even low millimolar range. This opens up the possibility of screening for smaller starting compounds 'chemical fragments' - which can then be built up into druglike molecules by adding optimal functionalities, ideally using the structure of the target protein derived from X-ray crystallographic data. This, argues Jhoti, should allow for "a more directed path from hit to the lead than the hit-and-miss process of refining larger molecules". Astex now has libraries of chemical fragments that bind in the low millimolar range, and already has examples of nanomolar lead compounds that were generated from millimolar hits.

Another company working with fragments is Graffinity Pharmaceuticals, in Heidelberg, Germany, which is taking advantage of the sensitivity of its optically based screening platform to test microarrays of chemical fragments. Technological challenges aside, another barrier to the development of fragment-based approaches is the generally held perception that high potency in binding to a target is a prerequisite for further development of a compound.

"There is a change of mindset needed in order to convince a chemist that a millimolar hit is going to be of use," says Jhoti. 